Proceedings

\title{
Research and Development of a Smart Internet-of-Things-Based System to Monitor and Prevent Household Gas Wastage +
}

\author{
Mohammad Monirujjaman Khan *, Md. Ibtida Fahim, Abrar Ahamed Habibullah, \\ Nowshin Tabassum and Aritra Sarker \\ Department of Electrical and Computer Engineering, North South University, Bashundhara, Dhaka1229, \\ Bangladesh; ibtida.fahim@northsouth.edu (M.I.F.); abrar.habibullah@northsouth.edu (A.A.H.); \\ nowshin.tabassum@northsouth.edu (N.T.); aritra.sarker@northsouth.edu (A.S.) \\ * Correspondence: monirujjaman.khan@northsouth.edu; Tel.: +88-017-7900-6296 \\ + Presented at the 1st International Electronic Conference on Applied Sciences, 10-30 November 2020; \\ Available online: https://asec2020.sciforum.net/.
}

Published: 10 November 2020

\begin{abstract}
In this paper, the development of a smart Internet of Things (IoT)-based system to monitor and prevent household gas wastage is proposed. An IoT-based gas wastage monitoring system was developed. The system needs to be integrated with the cooker. There are sensors integrated with the system that will find out if the cooker is being used for cooking purposes or not. If it is found that the cooker is not in use, there is automated switching-off technique in the system to turn off the supply of gas. The system also includes a cloud storage feature. With the help of this cloud storage system, the use of gas per day for each user can be monitored. This process will help to detect the misuse of natural gas of each user at the end of the day. The system has been tested and it is working fine. In the future, more features will be added to this system. This system will help prevent the wastage of natural gas and save the country from depleting its stores of it.
\end{abstract}

Keywords: Internet of Things; natural gas; cooking; cooker; wastage

\section{Introduction}

Natural gas is one of the most essential elements of our daily life. Natural gas is used for different areas, from households to industrial purposes. It is one of our main fuels for power plants and vehicles. Households use a significant amount of natural gas, as it is an essential component for cooking. In many countries, most of the households are interested in using natural gas for cooking rather than using electricity or other burner stoves. Furthermore, in Bangladesh, the majority of the households use natural gas for cooking purposes. People were using it for decades in an unorganized way. The majority of the people in Bangladesh are not aware of the critical demand forenergy. In our country, the amount of storage of natural gas is inadequate, and it is depleting rapidly. In Bangladesh, natural gas is used for many purposes. One of the most important uses of natural gas is in the household for cooking purposes. The major issue is that, in domestic cooking, the wastage of gas is very common here. Users sometimes forget to stop the cooker after they have finished of cooking, which causes the wastage of natural gas. The users of natural gas in domestic settings are not very serious about the wastage of gas. They are also not particularly aware of the consequences of the wastage the natural gas. It is very hard for the gas authority companies to monitor this type of wastage of gas and prevent the wastage of it. In this scenario, it is very urgent to monitor and stop the wastage of house hold gas that is used for cooking purposes [1]. 
Recently, research interest has grown in the area of the detection and prevention of gas leakage, and the monitoring and prevention of gas wastage [2-6]. Different techniques are applied in various articles. A number of research papers were published on gas leakage detection techniques [2-4]. In [5], thedesign and implementation of a simple electromechanical system to reduce domestic gas wastage and accidents in South Asia was presented. A smart ultrasonic device for vitro-ceramic cooker safety control was presented in [6]. However, to the knowledge of the authors of this paper, a smart Internet of Things (IoT)-based system to monitor and prevent household gas wastage has not previously been presented. In addition to that, in Bangladesh, such a system is not available. It is very important to develop an Internet of Things (IoT)-based system to monitor and prevent household gas wastage. The development of an IoT-based system to monitor and prevent house hold gas wastage is proposed and discussed in this paper. The system is very user friendly and cost effective. It costs only 10.41 US dollars, which is equivalent to 1383 Bangladeshi taka.

\section{Methodology}

In this section, the working principle of the whole system is described. Figure 1 shows the block diagram of the whole system, showing the function of each section. This system was designed through the combination of hardware and software. The main hardware components are an Arduino microcontroller, ultrasonic sensors, a flame sensor, and a motor. The software part is a cloud-based website: see Figure 1. In the proposed system, the ultrasonic and flame sensors are used to detect different actions. The ultrasonic sensor detects whether there is a cooking pot over the household gas burner. The flame sensor is used to detect the presence of the fire of the domestic cooker. The Arduino One UNO microcontroller is the mother component in this system. It is used as a controller unit for every sensor and action of the system. The Arduino UNO microcontroller receives the information from both sensors, and processes the data based on the data it determines in the action plan. The servo motor turns the gas supply on or off based on the decision. As mentioned earlier, a cloud-based feature is also included within the system. The Internet of Things data is stored and displayed in the website.

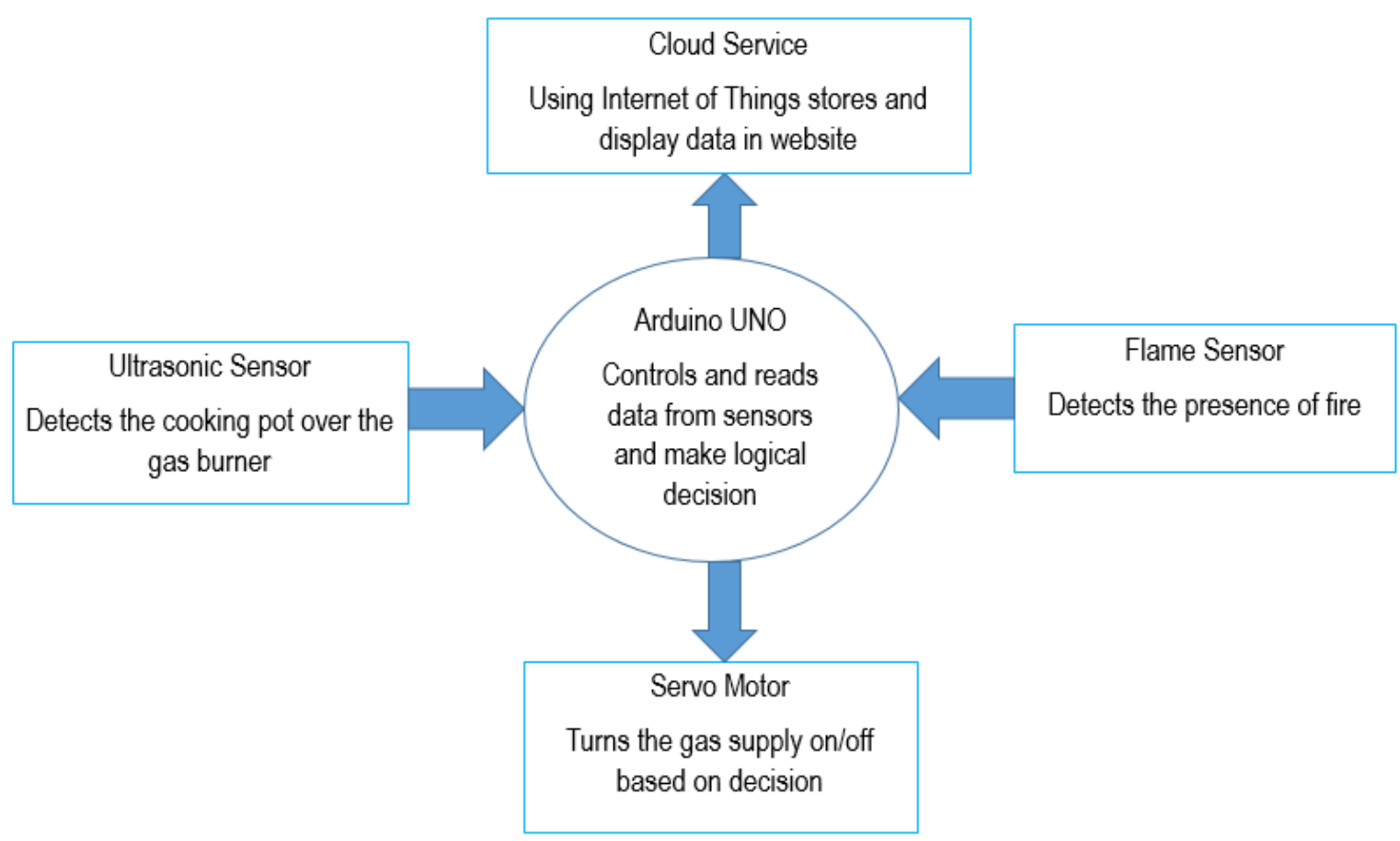

Figure 1. Block diagram of the whole system showing the function of each section.

Figure 2 shows the flow chart of the whole system with more details of the working principle of each section. The IoT-based gas monitoring and wastage system has to be placed on the cooker. The 
device needs to be switched on using the power button. Based on the reading of the ultrasonic sensor, the gas supply will be turned on or off. If any pot is detected on the cooker, the gas supply will be turned on, but if no pot is detected on the cooker, the gas supply will be turned off. If the flame sensor registers the presence of fire, and if the pot is not on the cooker, the gas supply will be turned off, and there will be no fire coming from the cooker: see Figure 2 for more details.

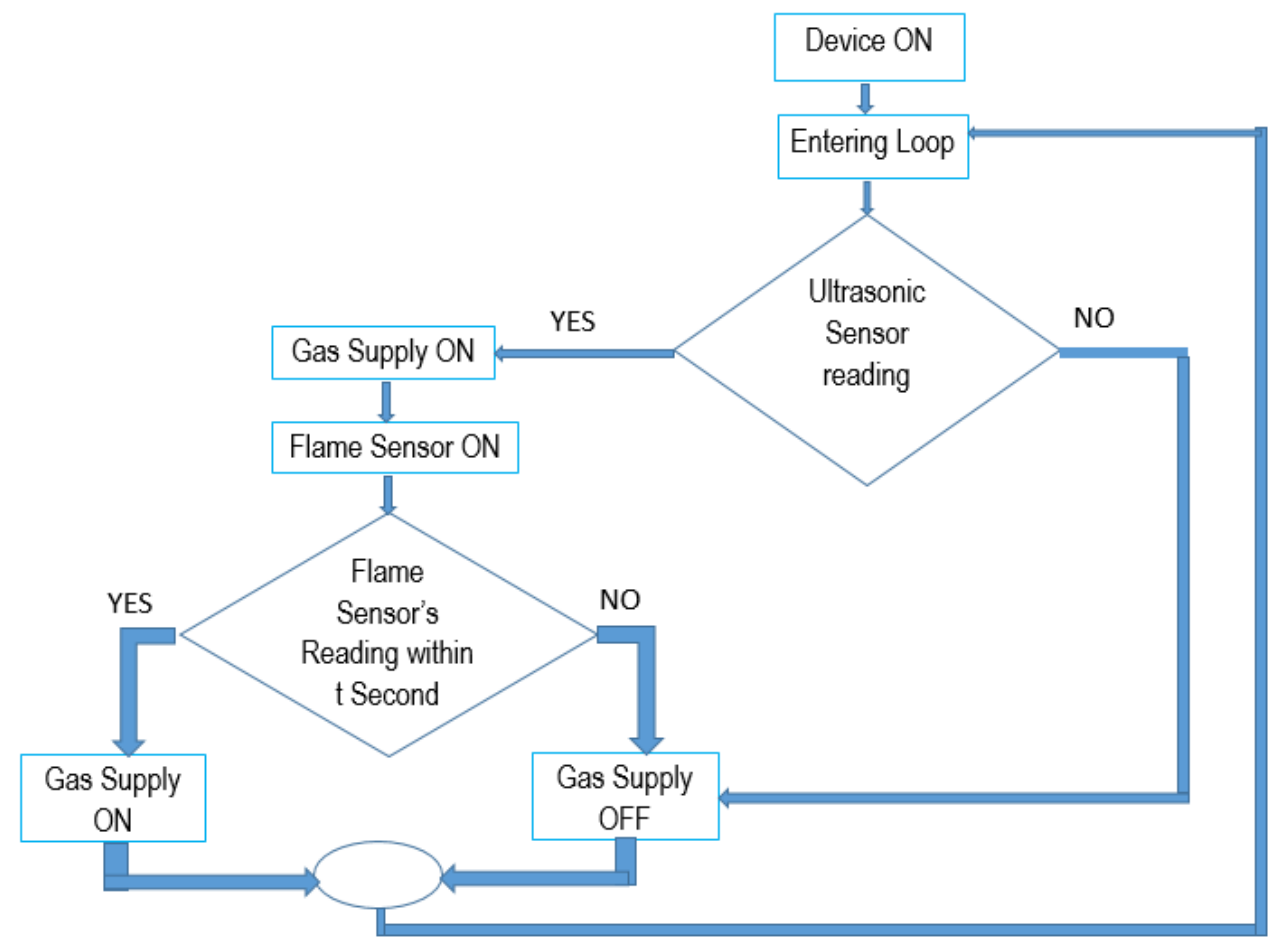

Figure 2. Flow chart of the working principle of the whole system, showing more details.

\section{Product Design and Analysis}

As was mentioned earlier, the IoT-basedhousehold gas monitoring and wastage prevention system was developed thorough the combination of a hardware and a software system. In order to design the system, the required pieces of hardware were an ultrasonic sensor, a flame sensor, an Arduino microcontroller, and a stepper motor. Table 1 lists the price of the hardware components in Bangladeshi taka and in US dollars. From Table 1, it can be observed that the cost of the hardware components is very cheap. In Bangladesh Taka, it can be noted that the cost is only 1383 Taka, which is equivalent to 10.40 US dollars. The system is very cost effective. More descriptions of the hardware components are provided in next paragraph.

Figure 3 demonstrates the ultrasonic sensor. This ultrasonic sensor module has transmitter and receiver parts, and it also has control unit. The ultrasonic sensor module works at very high frequencies,such as $40 \mathrm{KHz}$ sound waves [7]. The HC-SR04 ultrasonic sensor module has four pins: Voltage Common Collector VCC (power), Trig (Trigger), Echo (Receive), and GND Ground [8]. The HC-SR04 ultrasonic sensor module is basically a distance sensor which can detect the presence of any object within its working range. In this paper, anHC-SR04 ultrasonic sensor was utilized in order to detect the presence of any cooking pot on the cooker. The basic use and working principle of this sensor was described in an earlier section. 
Table 1. Cost of the hardware components.

\begin{tabular}{clccc}
\hline No & Name of the Components & Quantity & Price in BD Taka & Price in USD \\
\hline 1 & Ultrasonic Sensor & 1 & 118 & 1.39 \\
2 & Flame Sensor & 1 & 300 & 3.54 \\
3 & Arduino UNO Microcontroller & 1 & 465 & 5.48 \\
4 & Motor & 1 & 500 & 5.89 \\
& Total & 4 & 1383 & 10.41 \\
\hline
\end{tabular}

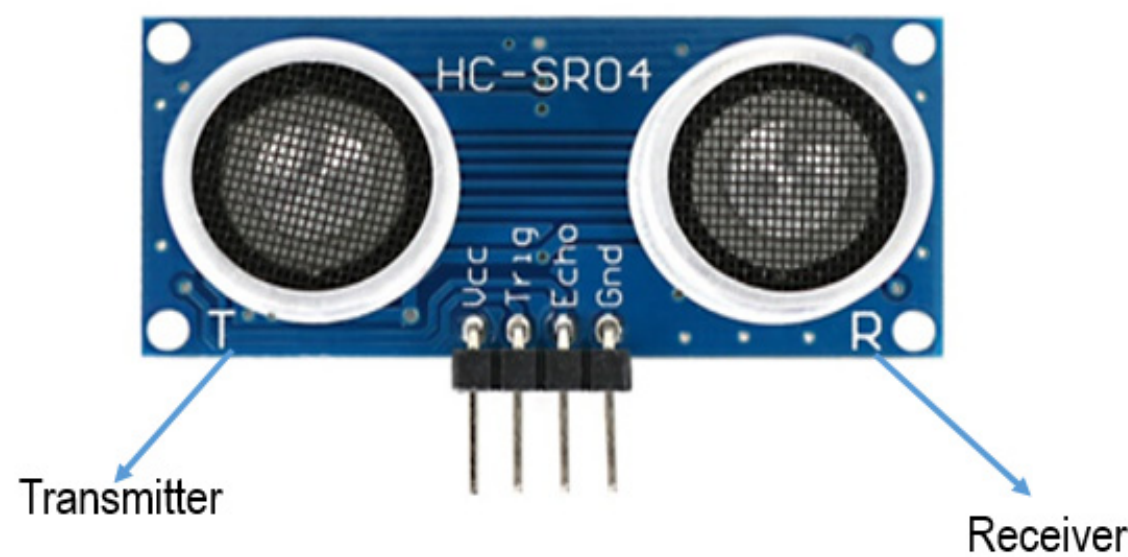

Figure 3. Ultrasonic Sensor [8].

Figure 4 shows the flame sensor that was used in this paper. A flame sensor is usually a detector that is designed for the detection of fire. This sensor/detector can be built with an electronic circuit using a receiver for, e.g., electromagnetic radiation. This sensor uses the infrared flame flash method. The flame sensor module has an Infrared (IR) receiver which can sense infrared radiation of a definite frequency that usually can be found in flame. This senor module has four pins: Pin 1(the VCC pin), Pin 2(GND), Pin 3 (the analog output, known as 'AOUT'), and Pin 4 (digital output, known as 'DOUT'). The detection angle of this flame sensor is 60 degrees [9]. This module is responsive to this flame range; it detects the flame after lighting the burner, and starts the processing part to run the algorithm, as described in the previous section.

Figure 5 shows the Arduino Uno microcontroller that was used in his paper. Arduino is an open-source platform that is usually used for building electronics projects. The Arduino consists of both a physical, programmable circuit board (often referred to as amicro controller) and a piece of software, or an IDE (Integrated Development Environment) that runs on the computer, which is used to write and upload computer code to the physical board. In this paper, the Arduino Uno microcontroller was used as the controller unit for each sensor and action. It works as a mother component for the proposed system. This Arduino Uno microcontroller maintains all of the sensors, processes the data, and takes action based on the data. This Arduino Uno microcontroller is programmed and works based on the algorithm. The operative principle of this component in this system was described in an earlier section. The Arduino Uno has 20 digital input/output pins (of which six can be used as Pulse Width Modulation PWM outputs, and six can be used as analog inputs), a $16 \mathrm{MHz}$ resonator, a Universal Serial Bus USB connection, a power jack, an in-circuit system programming (ICSP) header, and a reset button [10]. A stepper motor is an electromechanical device that converts electrical power into mechanical power. A high-torque stepper motor was attached tothe knob of the cooker for precision rotation [11]. This stepper motor turns the knob on/off according to the algorithm. 'Arduino IDE' was used for the general programming of the Arduino UNO microcontroller. A cloud storage feature was also integrated with the proposed system, with a view to monitor the usage of gas per day for each user. Basically, the data will be uploaded into a central database, and that will be shown on the website. This process will help to detect the misuse of natural gas for each user at the end of the day. 


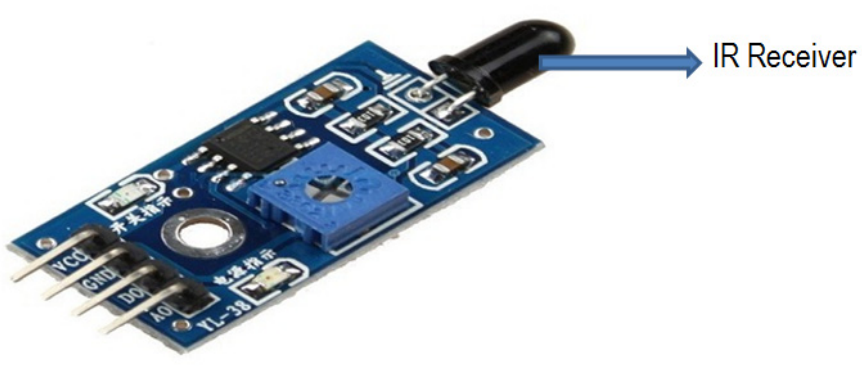

Figure 4. Flamesensor [9].

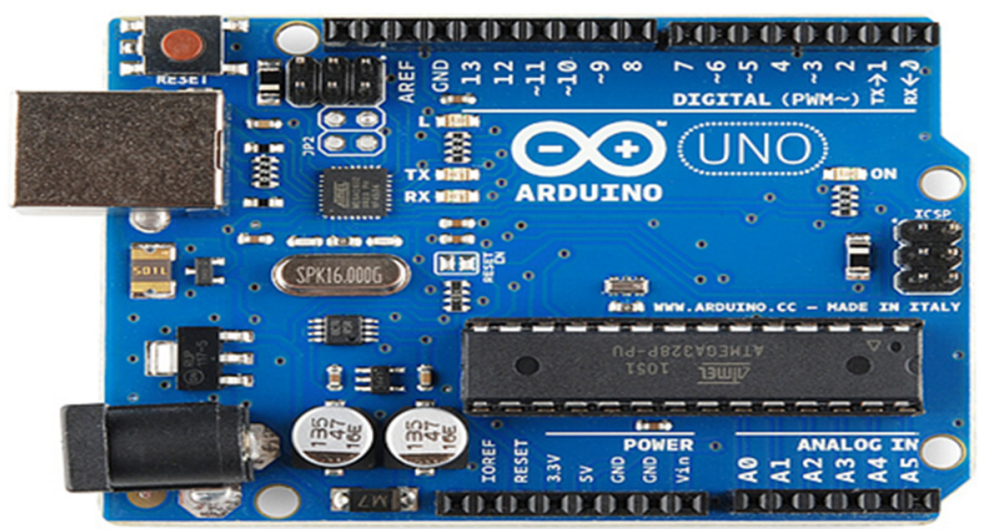

Figure 5. Arduino Uno microcontroller [10].

\section{Discussions}

The proposed system was developed and tested. The system worked well. Due to lack of page space, more technical details, results and analysis are not included in this paper. However, the authors of this paper are working to integrate more features into the proposed system. The authors plan to add more features to the cloud-based web system, and a mobile application is in development, in order to display the data on the mobile phone of the user. A gas leakage detection and alarm system are being merged with this system so that it can also detect gas leakages and control dangerous accidents. A Global System for Mobile Communications GSM module will be added with the new prototype so that, in case of any accident, the information of the incident can be sent to the emergency services, e.g., the ambulance and fire services. This product is innovative and, due to its low cost, it will be sustainable. However, more testing for greater numbers of user cases are needed for its real-life implementation as a commercial product. The authors are writing a journal paper in which all of the results will be summarized with more implementation and technical details. The article will be submitted to the MDPI journal for review.

\section{Conclusions}

The development and discussion of a smart Internet of Things (IoT)-based system to monitor and prevent household gas wastage were presented in this paper. The whole system is based on hardware and software. An Arduino UNO microcontroller, an ultrasonic senor, a flame sensor, and a stepper motor were used for the development of the hardware part of the proposed system. The design's working principles were described. The function of each segment of the proposed system was analyzed. A discussion of the required hardware and software were provided. The development cost of the system is very cheap. The cost of the hardware components is only 1383 Bangladeshi taka, which is equivalent to 10.41 US dollars. The device was developed and tested. The authors of the paper are working to introduce more features into the system, in order to make it smarter. After the integration of the new features into the system, it will be tested and implemented in real life scenarios. However, the application of this proposed system will be implemented in 
industrial areas, and the final device may be optimized for use with the industrial requirement. This system will stop the unnecessary usage of natural gas for cooking. This system will reduce the wastage of natural gas and save the country from depleting its stores of it. The country will benefit greatly from the use of the final product of this system.

Author Contributions: All authors contributed to the development of the system. The main author of the paper also wrote the manuscript. Input was also given by the co-authors. Currently, all of the co-authors are working on the further development and integration of the new features of the existing system with the guidance of main author. All authors have read and agreed to the published version of the manuscript.

Funding: This research received no external funding.

Acknowledgments: The authors of this papers would like to thank to the Department of Electrical and Computer Engineering of North South University. The development and integration work of this paper was carried out in the Engineering Laboratory in the Department of Electrical and Computer Engineering at North South University.

Conflicts of Interest: The authors declare no conflict of interest.

\section{References}

1. Wadud, Z.; Dey, H.S.; Kabir, A.; Khan, S.I. Modeling and forecasting natural gas demand in Bangladesh. Energy Policy 2011, 39, 7372-7380.

2. Shrivastava, A.; Prabhaker, R.; Kumar, R.; Verma, R. GSM based gas leakage detection system. Int. J. Emerg. Trends Electr. Electron. 2013, 3, 42-45.

3. Mahalingam, A., Naayagi, R.T.; Mastorakis, N.E. Design and implementation of an economic gas leakage detector. Recent Res. Appl. Electr. Comput. Eng.2012, 20-24.

4. Tsado, J.; Imoru, O.; Olayemi, S.O. Design and construction of a GSM based gas leak Alert system. IEEE Trans. 2014, 1, 2-6.

5. RHuq, R.M.; Hoque, A.M.; Chakraborty, P.; Jafar, I.B.; Rahman, K.H.; Hoque, A. Design and implementation of a simple electromechanical system to reduce domestic gas wastage and accidents in south-asia. In Proceedings of the 2012 Sixth International Conference on Sensing Technology (ICST), Kolkata, India, 18-21 December 2012; pp. 600-604.

6. Lázaro, A.; Serrano, I.; Guardado, F.; Herrero, R. Smart ultrasonic device for vitro-ceramic cooker safety control. In Proceedings of the1999 7th IEEE Inter-National Conference on Emerging Technologies and Factory Automation, Barcelona, Spain, 18-21 October 1999, Proceedings ETFA'99 (Cat. No. 99TH8467); Volume 1, pp. 565-570.

7. Ultrasonic Distance Sensor-HC-SR-04. Available online: https://www.sparkfun.com/products/15569 (accessed on 10 July 2020).

8. Flame Sensor Working and Its Applications. Available online: https://www.elprocus.com/flame-sensorworking-and-its-applications/\#: :text=A\%20sensor\%20which\%20is\%20most,known\%20as\%20a\%20flame \%20sensor.\&text=This\%20sensor\%20detects\%20flame\%20otherwise,certain $\% 20$ distance $\% 20$ from $\% 20$ the $\%$ 20flame (accessed on 10 July 2020).

9. What is an Arduino? Available online: https://learn.sparkfun.com/tutorials/what-is-an-arduino/all:: :text= Arduino\%20consists\%20of\%20both\%20a,code\%20to\%20the\%20physical\%20board(accessed on 10 July 2020).

10. Stepper Motor-Types, Advantages, and Applications. Available online: https://www.elprocus.com/steppermotor-types-advantages-applications/ (accessed on 11 July 2020).

11. Attia, A.H.; Ali, H.Y. Electronic Design of Liquefied Petroleum Gas Leakage Monitoring, Alarm, and Protection System Based on Discrete Components. Int. J. Appl. Eng. Res. 2016, 11, 9721-9726.

Publisher's Note: MDPI stays neutral with regard to jurisdictional claims in published maps and institutional affiliations. 